\title{
Endovascular treatment of symptomatic intracranial atherosclerotic disease
}

\author{
Jody L. Short ${ }^{1,2}$, Arshad Majid ${ }^{1,2}$ and Syed I. Hussain ${ }^{1,2 *}$ \\ 1 Department of Neurology, Michigan State University, East Lansing, MI, USA \\ 2 Sparrow Hospital Neurovascular Section, Lansing, MI, USA
}

Edited by:

Michael Chen, Rush University Medical Center, USA

\section{Reviewed by:}

Angelos A. Konstas, Massachusetts

General Hospital, USA

Weihai Xu, Peking Union Medical

College Hospital, Chinese Academy of

Medical Sciences, China

Aamir Badruddin, Medical College of

Wisconsin, USA

Muhammad A. Taqi, Medical College of

Wisconsin, USA

\section{*Correspondence:}

Syed I. Hussain, Vascular and

Interventional Neurology, Michigan

State University, 138 Service Road,

A217 Clinical Center, East Lansing, MI

48824, USA.

e-mail: syed.hussain@hc.msu.edu
Symptomatic intracranial atherosclerotic disease (ICAD) is responsible for approximately $10 \%$ of all ischemic strokes in the United States. The risk of recurrent stroke may be as high as 35\% in patient with critical stenosis $>70 \%$ in diameter narrowing. Recent advances in medical and endovascular therapy have placed ICAD at the forefront of clinical stroke research to optimize the best medical and endovascular approach to treat this important underlying stroke etiology. Analysis of symptomatic ICAD studies lead to the question that whether angioplasty and/or stenting is a safe, suitable, and efficacious therapeutic strategy in patients with critical stenoses that are deemed refractory to medical management. Most of the currently available data in support of angioplasty and/or stenting in high risk patients with severe symptomatic ICAD is in the form of case series and randomized trial results of endovascular therapy versus medical treatment are awaited. This is a comprehensive review of the state of the art in the endovascular approach with angioplasty and/or stenting of symptomatic ICAD.

Keywords: intracranial atherosclerosis, intracranial stenting, intracranial angioplasty, stent, angioplasty, stroke, Wingspan, Gateway

\section{INTRODUCTION}

Symptomatic intracranial atherosclerotic disease (ICAD) is responsible for approximately $10 \%$ of all ischemic strokes in the United States (Sacco et al., 1995; Qureshi et al., 2003). The prevalence of ICAD related ischemic stroke is higher in African American (17\%) and Hispanics (13\%) than among white Americans (9\%); however, it is highest in the Chinese population (33-37\%; Huang, 1997; Wong et al., 1998; White et al., 2005). Independent risk factors for ICAD are age, hypertension, and diabetes mellitus (Bae et al., 2007). Total serum cholesterol, HDL, and LDL may also be a risk factors for developing ICAD (Kostner et al., 1986; Suwanwela et al., 2006). Risk factors for recurrent ischemic stroke from ICAD include hypertension (systolic $\geq 140 \mathrm{mmHg}$ ), hyperlipidemia ( $\geq 200 \mathrm{mg} /$ dl), no alcohol consumption, and diabetes mellitus (Wong, 2003; Chaturvedi et al., 2007). Men may have a higher risk for developing ICAD particularly at younger ages (Passero et al., 1987; Moossy, 1993), but women appear to have a higher recurrent risk (Kasner et al., 2006). Angiotensin converting enzyme polymorphisms, plasma endostatin/vascular endothelial growth factor ratio, glutathione S-transferase omega-1 gene polymorphism, and plasma homocysteine levels are non-modifiable risk factors noted to be associated with ICAD. Sickle cell disease is an uncommon risk factor that can also be managed to reduce the risk of stroke. Associations of ICAD with diabetes mellitus, metabolic syndrome, Alzheimer's disease, aortic plaques, radiotherapy, and meningitis are less well documented (Suri and Johnston, 2009).

In the comparison of warfarin and aspirin for symptomatic intracranial arterial stenosis (WASID) trial, patients with symptomatic $50-99 \%$ intracranial stenosis had 12 and $11 \%$ risks of recurrent stroke in the symptomatic vascular territory at 1 year on aspirin or warfarin, respectively, and $17-20 \%$ at 2 years (Chimowitz et al., 2005). Patients with $70-99 \%$ stenosis had a recurrent stroke risk of $18 \%$ at 1 year compared to patients with $50-69 \%$ who had a 7\% risk of stroke at 1 year (Kasner et al., 2006). Additionally, time from qualifying event ( $\leq 17$ days) predicted risk of recurrent stroke (Kasner et al., 2006). Furthermore patients with intracranial stenosis who fail antithrombotic therapy were not at a higher risk for stroke than those who did not fail antithrombotic therapy (Turan et al., 2009). All of these analyses lead to the question that in patients with symptomatic severe ICAD defined as $\geq 70 \%$ stenosis is angioplasty and/or stenting a safe, effective, and viable treatment option? Currently, there is no data from a randomized controlled trial to guide clinicians toward an endovascular treatment of ICAD related ischemic strokes. Additionally there are no specific recommendations in the American Heart Association (AHA)/American Stroke Association (ASA) guidelines (Adams et al., 2008) for treatment or secondary prevention from ICAD related ischemic stroke. Most of the data currently available supporting angioplasty and stenting of ICAD is in the form of case series and there is no Level 1 evidence available to support endovascular therapy over medical management. The ongoing stenting and aggressive medical management for preventing recurrent stroke in intracranial stenosis (SAMMPRIS) trial is a randomized controlled trial that should help determine if intracranial stenting is superior to best medical management for symptomatic ICAD of 70-99\% (Derdeyn and Chimowitz, 2007).

The endovascular treatment of symptomatic ICAD has evolved with advances in technology. Originally balloons and stents designed for the coronary vasculature were used. 
Recently, stents designed specifically for the intracranial vasculature and the treatment of ICAD have been approved and are being used.

For the purpose of this review a literature search was performed using the keywords of intracranial angioplasty and/or stenting and cases series were identified in which either both or one of the modalities was used to treat symptomatic ICAD. We subsequently divided this review into categories based on the modality used either angioplasty and/or stenting and within the latter have reviewed the different stent types currently available.

\section{ENDOVASCULAR TREATMENT OF ICAD PRIMARY INTRACRANIAL ANGIOPLASTY}

Early studies with intracranial percutaneous transluminal angioplasty (PTA) reported a high risk of intraprocedural vessel rupture, vasospasm, dissection, or death in up to $33 \%$ of patients (Higashida et al., 1993; Terada et al., 1996; Callahan and Berger, 1997; Takis et al., 1997; Volk et al., 1997; Conners and Wojak, 1999; Marks et al., 1999). Angioplasty for symptomatic intracranial stenosis was first reported by Sundt et al. (1980). Subsequently several groups have reported successful PTA for ICAD defined as $<50 \%$ residual stenosis, with few complications in retrospective case series. Marks et al. (1999) reported 23 patients treated with PTA for symptomatic ICAD. Angioplasty resulted in reduced stenosis in 21 of 23 patients treated. In one patient, the lesion could not be crossed safely, and in the other patient balloon dilatations resulted in vessel rupture and death. During 3 years of follow-up the annual stroke rate was 3.2\%. Conners and Wojak (1999) have also reported a 9-year retrospective analysis of PTA for ICAD related ischemic stroke. Slower balloon inflation combined with balloon undersizing resulted in reduced complications, but occasionally yielded suboptimal angiographic results. Oversized balloons with rapid inflation were used in 12 patients and 9 of those patients (75\%) had angiographic dissections with 1 patient dying from a vessel occlusion. In comparison 50 patients were treated with undersized balloons and slower inflation times with a $14 \%$ risk of dissection all of which were asymptomatic.

Callahan and Berger (1997) treated 15 patients with ICAD related stroke who had failed warfarin therapy. Dilation was successful in all vessels, with residual stenosis averaging $<30 \%$; however, one patient died secondary to vessel rupture and another suffered an ischemic stroke in the pons secondary to perforator compromise. Residual stenosis occurred in $16 \%$ with no stenosis measuring over 70\%. Late restenosis occurred in $9 \%$ and were successfully retreated. One guidewire perforation occurred resulting in the death of a patient. Nasher et al. (2000) reported 20 patients treated with PTA for vertebrobasilar stenosis. Success rate was $86 \%$ with complete resolution of posterior circulation symptoms. One patient had an ischemic stroke during the procedure. There was a $14 \%$ rate of restenosis over 3-18 months.

A retrospective study was done to evaluate 36 patients with 37 symptomatic atherosclerotic intracranial stenosis who underwent primary balloon angioplasty after failing medical management (Marks et al., 2005). Thirty-four patients were available for follow-up at a mean of 53 months. The mean pretreatment stenosis was $84 \%$ before angioplasty and $43 \%$ after angioplasty. The periprocedural death and stroke rate was $8.3 \%$ (two deaths and one minor stroke). Two patients had strokes in the territory of angioplasty at 2 and 37 months after angioplasty. The annual stroke rate in the territory appropriate to the site of angioplasty was 3.4\%, and for those patients with a residual stenosis of $\geq 50 \%$ it was $4.5 \%$.

A Cochrane review of 79 articles (a total of 1999 cases) consisting of open-label case series with three or more cases of primary angioplasty was published in 2006 (Cruz-Flores and Diamond, 2006). The safety profile of the procedure showed an overall perioperative rate of stroke of $7.9 \%$, perioperative death of $3.4 \%$, and perioperative stroke or death of $9.5 \%$. Of these studies with a 1 -year follow-up the stroke rate at 1 year was $6 \%$.

All these descriptive studies show that the procedure is feasible though it carries a significant morbidity and mortality risk. Technical disadvantages such as dissection of the artery, vessel occlusion, immediate elastic recoil, and higher restenosis rates coupled with the vast amount of data from the coronary literature has rendered a somewhat inferior impression of balloon angioplasty alone compared to angioplasty with stenting; however, that being said the two modalities have not been compared head to head prospectively. Evidence from randomized controlled trials is needed to assess the safety of primary angioplasty and its effectiveness in preventing recurrent stroke.

\section{INTRACRANIAL ANGIOPLASTY AND STENT PLACEMENT}

Currently data exists for three different categories of stents in the treatment of ICAD: balloon expandable bare metal stents (BEBMS), drugeluting balloon expandable stents, and self-expanding stents. Stent systems can also be divided into those that are primarily designed for the coronary vasculature (Table 1) and those that have been specifically designed for the intracranial vasculature (Table 2 ).

\section{BALLOON EXPANDABLE BARE METAL STENTS}

Primary angioplasty without stent placement was occasionally complicated by dissection, acute vessel closure, and embolization (Higashida et al., 1993; Terada et al., 1996; Callahan and Berger, 1997; Takis et al., 1997; Volk et al., 1997; Conners and Wojak, 1999; Marks et al., 1999). Initially, BEBMS were used to an acute vessel dissection after angioplasty of a ICAD lesion; however, many of the coronary stents were rigid making them difficult to navigate into the intracranial circulation (Dorros et al., 1998). Several coronary stents were found to be sufficiently flexible to track into the intracranial circulation including the Gianturco-Rubin-2 stent (Phatouros et al., 1999), Palmaz-Schatz stent (Dorros et al., 1998), and the Arterial Vascular Engineering stent series which included the AVE S670stend and Guidant Tetramultilink stent (Morris et al., 1999; Phatouros et al., 1999).

Mori et al. $(1999,2000)$ treated 10 patients with 12 intracranial occlusive lesions with flexible BEBMS; however 2 lesions were not accessible. In six of eight vertebrobasilar and two distal internal carotid artery lesions GFX stents (Medtronics, Santa Rosa, CA, USA) were successfully deployed. Two distal internal carotid artery lesions were successfully stented with Multilink coronary stents (Guidant/Vascular Intervention, CA, USA). In the 10 lesions successfully treated, a pre-stent stenosis of $80 \%$ was reduced to $7 \%$. No periprocedural complications occurred and no ischemic events were reported in 11 months of follow-up. At 3 months all patients had follow-up angiograms which showed no restenosis. 
Table 1 | Summary of coronary stent studies used for treatment of intracranial stenosis.

\begin{tabular}{|c|c|c|c|c|c|}
\hline Study & Type & Subjects & 30-day outcomes & Long-term outcomes & Restenosis rates \\
\hline Mori et al. (2000) & Retrospective & 10 & No stroke or death & No ischemic events at 11 months & No restenosis at 3 months \\
\hline Gomez et al. (2000) & Retrospective & 12 & No stroke or death & 2 patients had a TIA at 6 months & $\begin{array}{l}2 / 12 \text { had F/U, } 1 \text { had basilar } \\
\text { occlusion at } 4 \text { months }\end{array}$ \\
\hline Mazighi et al. (2008) & Retrospective & $53^{*}$ & $10 \%$ stroke or death & $6 \%$ TIA or stroke at 2 years & $16 \%$ at 1 year \\
\hline Abou-Chebl et al. $(2005)^{* *}$ & Retrospective & 8 & 1 patient hadTIA & No stroke or TIAs at 11 months & No restenosis at 10 months \\
\hline Steinfort et al. (2007)** & Retrospective & 13 & $8 \%$ stroke & $8 \%$ stroke at 11 months & $\begin{array}{l}9 / 12 \text { had } F / U \text { with } 0 \% \\
\text { at } 5 \text { months }\end{array}$ \\
\hline Natarajan et al. $(2010)^{* * *}$ & Retrospective & 6 & $17 \%$ stroke & $0 \%$ at $4-10$ months & $0 \%$ at 6 months \\
\hline
\end{tabular}

${ }^{*}$ Only $76 \%$ of patients were treated with stents.

**Studies using drug-eluting stents.

***Second generation drug-eluting stent.

Table 2 | Symptomatic intracranial atherosclerosis studies using stents designed for intracranial use.

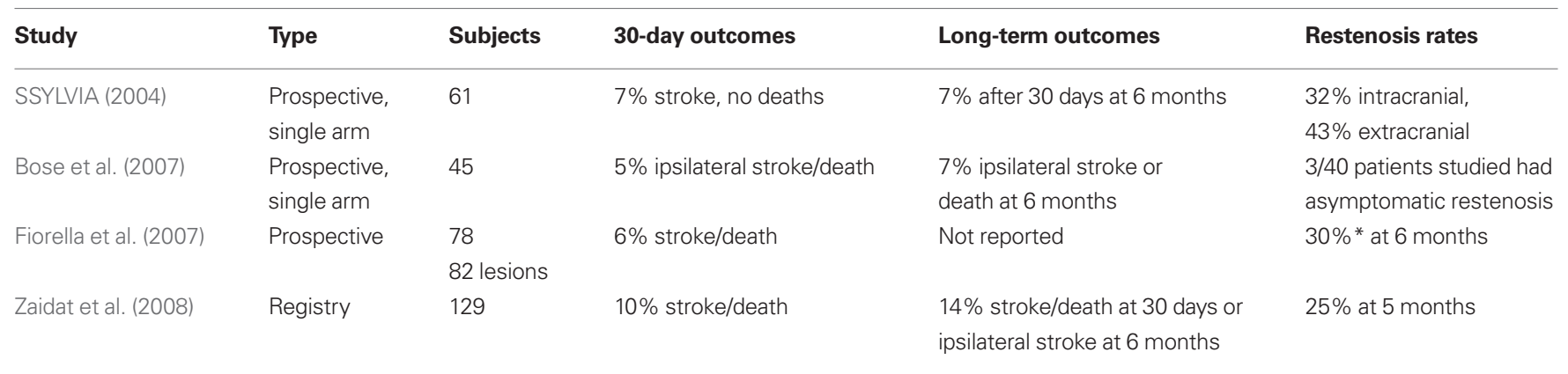

${ }^{*}$ Reported by Levy et al., (2007).

Gomez et al. (2000) treated 12 patients with symptomatic basilar artery stenosis with flexible BEBMS coronary stents (Microstent II or GFX, Advanced Vascular Engineering, Inc. or Multilink Duet, Guidant, Inc.). Stenosis was reduced from an average of $71-10 \%$. There were no deaths, stent thromboses, perforations, ruptures, or myocardial infarctions. One patient had non-specific symptoms, and another had a transient ischemic attack at a mean follow-up of 5.9 months.

Mazighi et al. (2008) retrospectively reviewed 53 consecutive symptomatic patients with 69 intracranial arterial lesions who were treated with coronary BEBMS. The technical success rate was $98.6 \%$, with a reduction of the median percent stenosis from 85 to $0 \%$. Stents were placed in $76.8 \%$. The 30 -day death or stroke rate was $10.1 \%$ with one death. With a median follow-up of 24 months, the transient ischemic attack or stroke rate reached $5.8 \%$. The restenosis rate at 1 year was $15.9 \%$ and was symptomatic in two patients.

The stenting of symptomatic atherosclerotic lesion in the vertebral or intracranial arteries (SSYLVIA) was a non-randomized, multicenter feasibility study that enrolled 61 patients with symptomatic disease (SSYLVIA Study Investigators, 2004). Forty-three patients with intracranial arteries (15 internal carotid, 5 middle cerebral, 1 posterior cerebral, 17 basilar, 5 vertebral) and 18 extracranial vertebral arteries [6 ostia, 12 proximal to the posterior inferior cerebellar artery (PICA)] were treated. In the SSYLVIA study, the NEUROLINK System (Guidant Corporation) was used. It was specifically designed for navigating intracranial vasculature by having only a few links connect the rings, rendering the stent flexible enough to navigate tortuous intracranial vessels. In the first 30 days, $6.6 \%$ had ischemic strokes and no deaths occurred. Of the 55 intracranial lesions treated 33 had stenosis measuring 70-99\% of which one patient had a stroke within the 30-day period. After 30 days, $7.3 \%$ of patients had recurrent ischemic strokes. Successful stent placement was achieved in $95 \%$ of cases. At 6 months, restenosis of $\geq 50 \%$ occurred in $32.4 \%$ intracranial arteries and $42.9 \%$ extracranial vertebral arteries. Thirty-nine percent of patients with recurrent stenoses were symptomatic. Post-procedure stenosis $>30 \%$, parent vessel diameter, and diabetes mellitus were all factors linked to restenosis. The NEUROLINK system however was not marketed past the trial as Guidant disbanded its neurovascular unit. Nevertheless this trial provided valuable data about the safety of stenting in the treatment of ICAD. Different from the prior BEBMS the NEUROLINK had been specifically designed with the intracranial vasculature in mind. Since its discontinuation there until very recently were no other BEBMS for ICAD. Micrus Corporation ${ }^{\circledR}$ (San Jose, CA, USA) most recently has designed the Pharos Vitesse Chrome Cobalt BEMS that is under study in Europe. 


\section{DRUG-ELUTING STENTS}

Some investigators have employed the use of drug-eluting stents (DES) to treat ICAD as have been used in the coronary vasculature. DES have a coating of antiproliferative material that inhibits neointimal hyperplasia. Steinfort et al. (2007) reported a review of 13 patients with symptomatic posterior circulation stenosis treated with pacilitaxel-coated stents. One patient suffered a periprocedural stroke, and nine patients had angiographic follow-up at a mean of 5.4 months with no significant in-stent stenosis. Qureshi et al. (2006) treated 18 patients with either a sirolimus-eluting stent $(n=14)$ or a paclitaxel-eluting stent $(n=4)$ for symptomatic stenosis $(\geq 70 \%$ or failed medical management). There was one major stroke and no death observed in the 1-month follow-up. Major stroke-free survival was $86 \%$ at 12 months. One symptomatic angiographic restenosis was observed during the follow-up period. Abou-Chebl et al. (2005) reviewed eight patients treated with DES with $>70 \%$ stenoses that had failed maximal medical therapy. Patients were pretreated with aspirin and clopidogrel and these were continued after the procedure for 1 year. Eight patients with intracranial lesions: internal carotid artery (3), middle cerebral (2), basilar (2), and vertebral artery (1) stenoses were successfully treated with 4 Cypher (Cordis Corp) and 4 Taxus (Boston Scientific) stents. The mean stenosis severity was reduced from 84.4 to $2.5 \%$. One patient had an intraprocedural retinal embolism, but there were no other complications. Over a mean follow-up of 11 months, patients had repeat angiography (5) or transcranial Doppler with or without CT angiography (3). None of the patients had clinical or significant angiographic restenosis. Likewise, Gupta et al. (2006) showed feasibility and safety by treating 62 symptomatic lesions, of which 26 were ICAD, with DES (Cypher or Taxus). Intracranial stenting was attempted in 29 but only successfully deployed in 26. At angiographic follow-up, 1 of 26 (5\%) intracranial DES and 2 of 36 extracranial DES were found to have restenosis $\geq 50 \%$ at a mean follow-up of 4 months. The previous DES studies used first generation stents with sirolimus or paclitaxel. Natarajan et al. (2010) reported six patients with symptomatic ICAD treated with a second generation DES that has greater deliverability. The Xience $\mathrm{V}$ everolimus-eluting stent (Abbott Vascular) was placed in six patients with $>70 \%$ stenosis. Average stenosis was reduced from 83 to $6 \%$. There was one post-intervention hemorrhage which required a craniectomy. Five of six patients had angiographic follow-up at 5-6 months with no restenosis.

There is still insufficient data to recommend the safe and effective use of DES in the intracranial vasculature and more study is required. The ongoing concerns with DES is the need for prolonged use of dual antiplatelet therapy with aspirin and clopidrogel which may increase the risk of hemorrhage especially in the brain and late restenosis.

\section{SELF-EXPANDING INTRACRANIAL STENTS}

The Wingspan stent system ${ }^{\otimes}$ (Boston Scientific) was the first selfexpanding intracranial stents (SEIS) to be approved for the treatment of ICAD in patients with medically refractory disease under a humanitarian device exemption (HDE) in August 2005. This was approved after being studied in a prospective, multicenter, singlearm intracranial stenting study for medically refractory patients in Europe and Asia (Bose et al., 2007). Forty-five patients with symptomatic ICAD who had $\geq 50 \%$ stenosis where included. The Wingspan stent system was used in all patients and consists of selfexpanding nitinol stent preloaded in a delivery catheter and a separately packaged Gateway PTA balloon catheter (Boston Scientific Corp; Figure 1). The 30-day composite ipsilateral stroke or death rate was $4.5 \%$, and $7 \%$ at 6 months. The degree of stenosis was reduced from an average of $75-32 \%$. Three patients had restenosis of $\geq 50 \%$ but were not symptomatic. There were no procedural instent thromboses or parent vessel dissections or perforations.

Subsequently the real life experience of this system has been recorded in two prospective registries. In the US Multicenter Experience with the Wingspan Stent System for the Treatment of Intracranial Atheromatous Disease all patients undergoing angioplasty and stenting using the Gateway balloon-Wingspan stent system were prospectively followed (Fiorella et al., 2007). During a 9-month period, treatment with the Wingspan stent system was attempted in 78 patients with 82 intracranial atheromatous lesions, of which 54 were $\geq 70 \%$ stenotic. Eighty-one of 82 lesions were successfully stented $(98.8 \%)$ during the first treatment session with the other treated only with angioplasty and stented at a later date. The mean pretreatment stenosis was $74.6 \%$, improving to $43.5 \%$ after balloon angioplasty and to $27.2 \%$ after stent placement. There were five $(6.1 \%)$ major periprocedural neurological complications, four of which ultimately led to patient death within 30 days of the procedure. Diffusion-weighted MRI sequences were done on 38 patients within $72 \mathrm{~h}$ of the procedure. Thirteen $(34.2 \%)$ had new ischemic lesions with only three being symptomatic.

In the NIH Registry on the use of the Wingspan Stent for Symptomatic 70-99\% Intracranial arterial stenosis 129 consecutive patients were enrolled (Zaidat et al., 2008). The technical success rate was $96.7 \%$. The mean pre-stent stenosis was $82 \%$ reduced to $20 \%$ after stenting. The frequency of any stroke, intracerebral hemorrhage, or death within 30 days or ipsilateral stroke beyond 30 days was $14 \%$ at 6 months. Any stroke or death occurred in eight patients $(6.2 \%)$ within 24 h of treatment. Fifty-two patients (40\%) had follow-up cerebral angiograms at a mean of 4.8 months. The mean residual stenosis was $29 \%$, with 13 patients $(25 \%)$ having restenosis $\geq 50 \%$, and 2 patients had ischemic stokes with both attributed to stent occlusions.

The Wingspan SEIS is currently under study in SAMMPRIS to compare stenting with aggressive medical management.

\section{COMPARISON OF BEBMS/DES TO SEIS}

Gröschel et al. (2009) conducted a systematic review 31 studies dealing with 1177 intracranial stenting procedures from 1998 to 2008. Ninety-eight percent were performed in patients with a

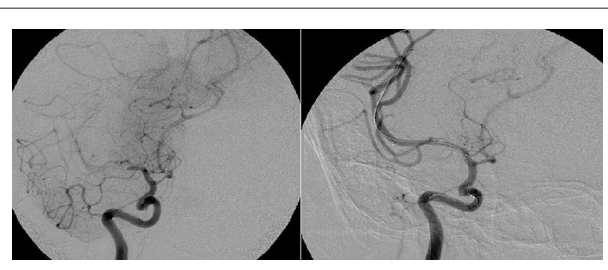

FIGURE 1 | Right middle cerebral artery stenosis treated with SEIS/ Wingspan stent. 
symptomatic high-grade (mean: 78\%) intracranial stenosis. The median technical success rate was $96 \%$. The periprocedural minor or major stroke and death rates ranged from 0 to $50 \%$ with a median of $7.7 \%$. Periprocedural complications were significantly higher in the posterior $(12.1 \%)$ versus the anterior circulation $(6.6 \%)$, but did not differ between patients treated with a balloon-mounted versus those who had been treated with a self-expandable stent(s). However restenosis $\geq 50 \%$ occurred more frequently after the use of a self-expandable stent (17.4\%, mean follow-up of 5.4 months) than a balloon-mounted stent (13.8\%, mean follow-up of 8.7 months). This has been attributed to the higher amount of radial force applied to the vessel by BEBMS and that most SEIS are deployed after a submaximal angioplasty.

A comparison of stent design between coronary and cerebral stents can be seen in Figure 1.

\section{RESTENOSIS AFTER ANGIOPLASTY AND STENTING}

Albuquerque et al. (2008) described in-stent restenosis (ISR) as $>50 \%$ stenosis within or immediately adjacent (within $5 \mathrm{~mm}$ ) to the implanted stent(s) and $>20 \%$ absolute luminal loss. Imaging follow-up (3-15.5 months) was available for 127 intracranial stenotic lesions treated with the Wingspan stent system. Forty-one lesions (32\%) developed either ISR (28\%) or complete stent occlusion (4\%) after treatment. When restenotic lesions were characterized using the modified classification system, 25 of 41 (61.0\%) were focal lesions involving $<50 \%$ of the length of the stented segment: three were Type IA (focal stenosis involving one end of the stent), 21 were Type IB (focal intrastent stenosis involving a segment completely contained within the stent), and one was Type IC (multiple non-contiguous focal stenoses). Eleven lesions (26.8\%) demonstrated diffuse stenosis ( $>50 \%$ of the length of the stented segment): nine were Type II with diffuse intrastent stenosis (completely contained within the stent) and two were Type III with proliferative in-stent stenosis (extending beyond the stented segment). Five stents were completely occluded at follow-up (Type IV). Of the 36 ISR lesions, 16 were less severe or no worse than the original lesion with respect to severity of stenosis or length of the segment involved. Twenty lesions were more severe than the original lesion. Supraclinoid internal carotid artery and middle cerebral artery lesions have a propensity to develop more severe post-treatment stenosis.

Levy et al. (2007) reported follow-up imaging (average duration, 5.9 months) available for 84 lesions treated with the Wingspan stent (78 patients). Follow-up examinations consisted of 65 conventional angiograms, 17 computed tomographic angiograms, and two magnetic resonance angiograms. ISR was documented in 25 and complete thrombosis in 4 patients. Two of the four patients with stent thrombosis had lengthy lesions requiring more than one stent to bridge the diseased segment. ISR was more frequent in the anterior circulation $(42 \%)$ than the posterior circulation (13\%). Eight patients were symptomatic (four with stroke, four with transient ischemic attack) and 15 were retreated. Of these, four were complicated by clinically silent in-stent dissections, two of which required the placement of a second stent. One patient had a post-procedural reperfusion hemorrhage.

The ISR rate with the Wingspan stent is higher in the multicenter experience Levy and colleagues (Fiorella et al., 2007; 30\%) than in the original Wingspan study (Bose et al., 2007; 15\%), but comparable to the NIH Wingspan registry (Zaidat et al., 2008; $25 \%)$. Typically ISR is asymptomatic (76\%) but it can cause neurological symptoms (Levy et al., 2007). Thirty-six patients in the registry experienced in ISR after PTA and stenting with Wingspan. Of these patients, 29 (80.6\%) have undergone target lesion revascularization with either angioplasty alone $(n=26)$ or angioplasty with restenting $(n=3)$. Restenting was performed for in-stent dissections that occurred after the initial angioplasty. Of the 29 patients undergoing target lesion revascularization, 9 required $\geq 1$ interventions for recurrent ISR, for a total of 42 interventions. One major complication, a post-procedural reperfusion hemorrhage, was encountered in the periprocedural period $(2.4 \%$ per procedure; $3.5 \%$ per patient). Angiographic follow-up was available for 22 of 29 patients after target lesion revascularization. Eleven of 22 (50\%) demonstrated recurrent ISR at follow-up angiography. Nine patients had multiple retreatments for recurrence. Recurrent lesions were more common in the anterior circulation.

In-stent restenosis in most instances is asymptomatic and therefore its management and the need for retreatment with angioplasty and/or stenting is not clear. However most operators feel the need to retreat if the ISR causes a stenosis that is worse than the original lesion or the patient becomes symptomatic. Further study is required to identify patients at risk, potentially modifiable risk factors and methods of treatment of ISR after stenting of ICAD.

\section{MEDICAL MANAGEMENT COMPARED TO ENDOVASCULAR TREATMENT}

Patients from the WASID trial (254) and from the NIH Wingspan multicenter stent registry (158) were matched to determine the differential rate of stroke or death within 30 days or ipsilateral stroke beyond 30 days (Jarvis et al., 2008). They were divided into two categories of 50-69\% and 70-99\% stenosis. The primary event rates in WASID patients with $70-99 \%$ stenosis was 7 and $16 \%$, respectively; the comparable rates in the Wingspan group were 10 and $13 \%$. There was no difference in the 50-69\% groups. As mentioned previously, SAMMPRIS is an ongoing trial comparing stenting combined with best medical management to best medical management alone.

\section{PRIMARY ANGIOPLASTY COMPARED TO ANGIOPLASTY WITH STENT PLACEMENT}

Siddiq et al. (2008) retrospectively analyzed the clinical and angiographic data of 190 patients treated with 95 primary angioplasty procedures and 98 intracranial stent placements in three tertiary care centers. Fourteen procedures in the angioplasty-treated group (15\%) and four in the stent-treated group (4\%) had significant post-operative residual stenosis. There were 3 periprocedural deaths $(1.5 \%), 1$ in the angioplasty group (1\%) and 2 in the stent-treated group $(2.0 \%)$ and 14 periprocedural strokes (7.3\%), 7 periprocedural strokes in each group (7.4 and 7.1\%). Angiographic follow-up was available for 134 procedures (66 angioplasty-treated and 68 stent-treated cases). Forty-eight patients had restenosis (25 of 66 angioplasties, 23 of 68 stents). Restenosis-free survival at 12 months was $68 \%$ for the angioplasty-treated group and $64 \%$ for the stenttreated group. There was no difference in follow-up survival (stroke, or stroke and/or death) between the angioplasty-treated and the stent-treated groups at 2 years. 


\section{PERIPROCEDURAL MANAGEMENT}

Based on the coronary intervention literature, practitioners are continuing dual antiplatelet agents for a total of 3 months after intracranial stent placement, although the range is $4-12$ weeks (Schleinitz et al., 2004). Clopidogrel $75 \mathrm{mg}$ daily with aspirin (81 or $325 \mathrm{mg}$ ) for at least $72 \mathrm{~h}$ before the intervention is recommended. If not possible, a loading dose of clopidogrel $300 \mathrm{mg}$ within 6-24 h or $600 \mathrm{mg}$ within $6 \mathrm{~h}$ is reasonable (Bertrand et al., 2000). In patients allergic to clopidogrel, ticlopidine $250 \mathrm{mg}$ daily or a 500-mg loading dose is a reasonable alternative (Levy et al., 2007). DES for coronary intervention require 1 year of dual antiplatelet therapy (Schleinitz et al., 2004), which is also reasonable for DES in the cerebral vasculature (Qureshi et al., 2009). Additionally a very important factor is that stenting should not be a substitute for aggressive risk factor control and continued attention to control all modifiable risk factors needs to be paid. This should include aggressive blood pressure, diabetes, and dyslipidemia management per stroke guidelines (Adams et al., 2008).

Complications to be considered around the time of angioplasty and stenting can be of varying severity (Jiang et al., 2007; Samaniego et al., 2009). Dangerous life threatening complications with severe morbidity and mortality are mainly from the risk of dissection and rupture of the parent vessel. Acute stent thrombosis can lead to occlusion of the stent and vessel predisposing to a malignant infarct and edema within the vessels vascular territory and usually occurs within a setting of suboptimal antiplatelet therapy. Furthermore acute to subacute revascularization with stenting after stroke can predispose to secondary hemorrhage within the ischemic bed.

When stenting is undertaken in perforator rich arteries there is a risk of the "snow plow" effect of pushing atheroma into the ostia of perforators and consequently causing a perforator stroke (PS; Figure 2). In a study by Jiang et al. (2006) using BMS a 3\% PS rate was identified. The presence of preprocedural PS was associated with a higher likelihood of developing a new PS. This is in contrast to a study by Leung et al. (2009) which using SEIS (Wingspan ${ }^{\mathrm{TM}}$ ) had a $0 \%$ PS rate by MRI imaging over a 4 -month period. It is felt that the submaximal $<80 \%$ angioplasty used with SEIS is less likely to push plaque into perforators. However there are concerns with the continued outward radial force exerted by SEIS may cause more neointimal hyperplasia and restenosis which ultimately may cause delayed PSs.

\section{REFERENCES}

Abou-Chebl, A., Bashir, Q., and Yadav, J. S. (2005). Drug-eluting stents for the treatment of intracranial atherosclerosis: initial experience and midterm angiographic follow-up. Stroke 36, e165-e168.

Adams, R. J., Albers, G., Alberts, M. J., Benavente, O., Furie, K., Goldstein, L. B., Gorelick, P., Halperin, J., Harbaugh, R., Johnston, S. C., Katzan, I., KellyHayes, M., Kenton, E. J., Marks, M., Sacco, R. L., and Schwamm, L. H. (2008). Update to the AHA/ASA recommendations for the prevention of stroke in patients with stroke and transient ischemic attack. Stroke 39, 1647-1652.

Albuquerque, F. C., Levy, E. I., Turk, A. S., Niemann, D. B., Aagaard-Kienitz, B., Pride, G. L.Jr., Purdy, P.D., Welch, B. G., Woo, H.H., Rasmussen, P.A., Hopkins, L. N., Masaryk, T. J., McDougall, C. G., and Fiorella, D. J. (2008). Angiographic patterns of Wingspan in-stent restenosis. Neurosurgery 63, 23-28.

Bae, H. J., Lee, J., Park, J. M., Kwon, O., Koo, J. S., Kim, B. K., and Pandey, D. K. (2007). Risk factors of intracranial cerebral atherosclerosis among asymptomatics. Cerebrovasc. Dis. 24, 355-360.

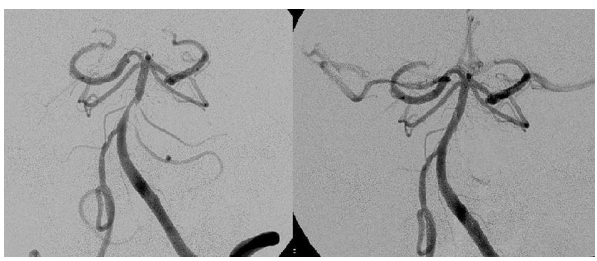

FIGURE 2 | Basilar stenosis treated with DES. Note absent perforator/side branches post-stenting

For these reasons endovascular therapy of symptomatic ICAD is best adopted through a multidisciplinary approach with stroke neurology, neuroradiology, neurosurgery, and neurointerventionalists. Careful patient selection is important paying special attention to the risk of hemorrhagic and thromboembolic complications that maybe encountered. Post-procedural neurointensive care and astute nursing care are paramount to help recognize these potential complications in a timely fashion.

In the absence of randomized data we cannot make a strong argument for angioplasty and stenting but it is an option that is available to patients in whom medical risk factors have already been maximally controlled and continue to have ongoing ischemic symptoms. Stenting appears to be more durable than angioplasty alone as we have discussed during this review however host factors such as difficult anatomy, vessel tortuosity, heavily calcified lesions, and the safety or tolerability of prolonged antiplatelet therapy may preclude stent placement and in our experience angioplasty alone is still an option in those scenarios.

Amongst the types of stents there is no randomized data comparing BMS to SEIS. The initial excitement with SEIS has been quelled by restenosis rates however on the other hand the trackability and deliverability of BMS seems to be inferior to that of SEIS.

\section{CONCLUSION}

Technological advances over the last decade have made intracranial angioplasty and stenting a possible therapeutic option for patients with severe symptomatic ICAD. However at this point there is lack of evidence for its long-term efficacy when compared to medical therapy. Results of randomized trials comparing stenting to medical therapy are much awaited to see if endovascular management will have a more concrete role in the secondary prevention of stroke.

Bertrand, M. E., Rupprecht, H. J., Urban, P., and Gershlick, A.H. (2000). Doubleblind study of the safety of clopidogrel with and without a loading dose in combination with aspirin compared with ticlopidine in combination with aspirin after coronary stenting: the clopidogrel aspirin stent international cooperative study (CLASSICS). Circulation 102, 624-629.

Bose, A., Hartmann, M., Henkes, H., Liu, H. M., Teng, M. M., Szikora, I., Berlis, A., Reul, J., Yu, S. C., Forsting, M., Lui, M., Lim, W., and Sit, S. P. (2007). A novel, self-expanding, nitinol stent in medically refractory intracranial atherosclerotic stenosis: the Wingspan study. Stroke 38, 1531-1537.

Callahan, A. S., and Berger, B. L. (1997). Balloon angioplasty of intracranial arteries for stroke prevention. J. Neuroimaging 7, 232-235.

Chaturvedi, S., Turan, T. N., Lynn, M. J., Kasner, S. E., Romano, J., Cotsonis, G., Frankel, M., and Chimowitz, M. I. (2007). Risk factor status and vascular events in patients with symptomatic intracranial stenosis. Neurology 69, 2063-2068.

Chimowitz, M. I., Lynn, M. J., HowlettSmith, H., Stern, B. J., Hertzberg, V. S., Frankel, M. R., Levine, S. R., 
Chaturvedi, S., Kasner, S. E., Benesch, C. G., Sila, C. A., Jovin, T. G., and Romano, J. G. (2005). Comparison of warfarin and aspirin for symptomatic intracranial arterial stenosis. N. Engl. J. Med. 352, 1305-1316.

Conners, J. J., and Wojak, J. C. (1999). Percutaneous transluminal angioplasty for intracranial atherosclerotic lesions: evolution of technique and short-term results. J. Neurosurg. 91, 415-423.

Cruz-Flores, S., and Diamond, A. L. (2006). Angioplasty for intracranial artery stenosis. Cochrane Database Syst. Rev. 3, CD004133.

Derdeyn, C. P., and Chimowitz, M. I. (2007). Angioplasty and stenting for atherosclerotic intracranial stenosis: rationale for a randomized clinical trial. Neuroimaging Clin. N. Am. 17, 355-363.

Dorros, G., Cohn, J. M., and Palmer, L. E. (1998). Stent deployment resolves a petrous carotid artery angioplasty dissection. Am. J. Neuroradiol. 19, 392-394.

Fiorella, D., Levy, E. I., Turk, A. S., Albuquerque, F. C., Niemann, D. B., Aagaard-Kienitz, B., Hanel, R.A., Woo, H., Rasmussen, P. A., Hopkins, L. N., Masaryk, T. J., and McDougall, C. G. (2007). US multicenter experience with the Wingspan stent system for the treatment of intracranial atheromatous disease: periprocedural results. Stroke 38, 881-887.

Gomez, C. R., Misra, V. K., Liu, M. W., Wadlington, V. R., Terry, J. B., Tulyapronchote, R., and Campbell, M. S. (2000). Elective stenting of symptomatic basilar artery stenosis. Stroke 31, 95-99.

Gröschel, K., Schnaudigel, S., Pilgram, S. M., Wasser, K., and Kastrup, A. (2009). A systematic review on outcome after stenting for intracranial atherosclerosis. Stroke 5, e340-e347.

Gupta, R., Al-Ali, F., Thomas, A. J., Horowitz, M.B., Barrow, T., Vora, N.A., Uchino, K., Hammer, M. D., Wechsler, L. R., and Jovin, T. G. (2006). Safety, feasibility, and short-term follow-up of drug-eluting stent placement in the intracranial and extracranial circulation. Stroke 37, 2562-2566.

Higashida, R. T., Tsai, F. Y., Halbach, V. V., Dowd, C. F., and Hieshima, G. B. (1993). Cerebral percutaneous transluminal angioplasty. Heart Dis. Stroke 2, 497-740.

Huang, Y. (1997). Vascular lesions in Chinese patients with transient ischemic attacks. Neurology 48, 524-525.

Jarvis, A. L., Chimowitz, M., and Lynn, M. J. (2008). NIH multicenter Wingspan intracranial stent study group. Outcome of patients with 50-99\% intracranial stenosis and TIA or stroke on antithrombotic therapy treated medically vs. stenting. Presented at the 60th Annual Meeting of the American Academy of Neurology, Chicago, IL.

Jiang, W. J., Du, B., Leung, T. W., Xu, X. T., Jin, M., and Dong, K. H. (2007). Symptomatic intracranial stenosis: cerebrovascular complications from elective stent placement. Radiology 243, 188-197.

Jiang, W. J., Srivastava, T., Gao, F., Du, B., Dong, K. H., and Xu, X. T. (2006). Perforator stroke after elective stenting of symptomatic intracranial stenosis. Neurology 66, 1868-1872.

Kasner, S. E., Chimowitz, M. I., Lynn, M. J., Howlett-Smith, H., Stern, B. J., Hertzberg, V. S., Frankel, M. R., Levine, S. R., Chaturvedi, S., Benesch, C. G., Sila, C. A., Jovin, T. G., Romano, J. G., and Cloft, H. J. (2006). Predictors of ischemic stroke in the territory of a symptomatic intracranial arterial stenosis. Circulation 113, 555-563.

Kostner, G. M., Marth, E., Pfeiffer, K. P., and Wege, H. (1986). Apolipoproteins AI, AII and HDL phospholipids but not APO-B are risk indicators for occlusive cerebrovascular disease. Eur. Neurol. 25, 346-354.

Leung, T. W., Yu, S. C., Lam, W. W., Chan, A. Y., Lau, A. Y., and Wong, L. K. (2009). Would self-expanding stent occlude middle cerebral artery perforators? Stroke 40, 1910-1912.

Levy, E. I., Turk, A. S., Albuquerque, F. C., Niemann, D. B., Aagaard-Kienitz, B., Pride, L., Purdy, P., Welch, B., Woo, H., Rasmussen, P. A., Hopkins, L. N., Masaryk, T. J., McDougall, C. G., and Fiorella, D. J. (2007). Wingspan in-stent restenosis and thrombosis: incidence, clinical presentation, and management. Neurosurgery 61, 644-651.

Marks, M. P., Marcellus, M., Norbash, A. M., Steinberg, G. K., Tong, D., and Albers, G.W. (1999). Outcome of angioplasty for atherosclerotic intracranial stenosis. Stroke 30, 1065-1069.

Marks, M. P., Marcellus, M. L., Do, H. M., Schraedley-Desmond, P. K., Steinberg, G. K., Tong, D. C., and Albers, G. W. (2005). Intracranial angioplasty without stenting for symptomatic intracranial atherosclertotic stenosis: long term follow-up. Am. J. Neuroradiol. 3, 525-530.

Mazighi, M., Yadav, J.S., and Abou-Chebl, A. (2008). Durability of endovascular therapy for symptomatic intracranial atherosclerosis. Stroke 39, 1766-1769.

Moossy, J. (1993). Pathology of cerebral atherosclerosis. Influence of age, race, and gender. Stroke 24, I22-I23, I31-I32.

Mori, T., Kazita, K., Chokyu, K., Mima, T., and Mori, K. (2000). Short-term arteriographic and clinical outcome after cerebral angioplasty and stenting for intracranial vertebrobasilar and carotid atherosclerotic occlusive disease. Am. J. Neuroradiol. 21, 249-254

Mori, T., Kazita, K., Seike, M., Nojima, Y., and Mori, K. (1999). Successful cerebral artery stent placement for total occlusion of the vertebrobasilar artery in a patient suffering from acute stroke. Case report. J. Neurosurg. 90 955-958.

Morris, P. P., Martin, E. M., Regen, J., and Braden, G. (1999). Intracranial deployment of coronary stents for symptomatic atherosclerotic disease. Am. J. Neuroradiol. 20, 1688-1694.

Nasher, H. C., Henkes, H., Weber, W. Berg-Dammer, E., Yousry, T. A., and Kühne, D. (2000). Intracranial vertebrobasilar stenosis: angioplasty and follow-up. Am. J. Neuroradiol. 21 , 1293-1301.

Natarajan, S. K., Ogilvy, C. S., Hopkins, L. N., AH Siddiqui, EI Levy. (2010). Initial experience with an everolimuseluting, second generation drug-eluting stent for treatment of intracranial atherosclerosis. J. Neurointerv. Surg. 2, 104-109.

Passero, S., Rossi, G., Nardini, M., Bonelli, G., D’Ettorre, M., Martini, A., Battistini, N., Albanese, V., Bono, G., and Brambilla, G. L. (1987). Italian multicenter study of reversible cerebral ischemic attacks. Part 5. Risk factors and cerebral atherosclerosis. Atherosclerosis 63, 211-224.

Phatouros, C. C., Higashida, R. T., Malek, A. M., Smith, W. S., Mully, T. W., DeArmond, S. J., Dowd, C. F., and Halbach, V. V. (1999). Endovascular stenting of an acutely thrombosed basilar artery: technical case report and review of the literature. Neurosurgery 44, 667-673.

Qureshi, A. I., Feldmann, E., Gomez, C. R., Johnston, S. C., Kasner, S. E., Quick, D. C., Rasmussen, P. A., Suri, M. F. Taylor, R. A., and Zaidat, O. O. (2009). Intracranial atherosclerotic disease: an update. Ann. Neurol. 66, 730-738.

Qureshi, A. I., Kirmani, J. F., Hussein, H. M., Harris-Lane, P., Divani, A. A., Suri, M. F., Janjua, N., and Alkawi, A. (2006). Early and intermediate-term outcome with drug-eluting stents in high risk patients with symptomatic intracranial stenosis. Neurosurgery 5 , 1044-1051.

Qureshi, A. I., Ziai, W. C., Yahia, A. M. Mohammad, Y., Sen, S., Agarwal, P., Zaidat, O. O., Suarez, J. I., and Wityk, R. J. (2003). Stroke-free survival and its determinants in patients with symptomatic vertebrobasilar stenosis: a multicenter study. Neurosurgery 52 , 1033-1039; discussion 1039-1040.

Sacco, R. L., Kargman, D. E., Gu, Q., and Zamanillo, M. C. (1995). Raceethnicity and determinants of intracranial atherosclerotic cerebral infarction: the Northern Manhattan stroke study. Stroke 27, 1974-1980.

Samaniego, E. A., Hetzel, S., Thirunarayanan, S., Aagaard-Kienitz, B., Turk, A. S., and Levine, R. (2009). Outcome of symptomatic intracranial atherosclerotic disease. Stroke 40 2983-2987.

Schleinitz, M. D., Olkin, I., and Heidenreich, P. A. (2004). Cilostazol, clopidogrel or ticlopidine to prevent sub-acute stent thrombosis: a metaanalysis of randomized trials. Am. Heart J. 148, 990-997.

Siddiq, F., Vazquez, G., Memon, M. Z., Suri, M. F., Taylor, R. A., Wojak, J. C., Chaloupka, J. C., and Qureshi, A. I. (2008). Comparison of primary angioplasty with stent placement for treating symptomatic intracranial atherosclerotic disease: a multicenter study. Stroke 39, 2505-2510.

SSYLVIA Study Investigators. (2004) Stenting of symptomatic atherosclerotic lesions in the vertebral or intracranial arteries (SSYLVIA): study results. Stroke 35, 1388-1392.

Steinfort, B., Ng, P. P., Faulder, K., Harrington, T., Grinnell, V., Sorby, W., and Morgan, M. K. (2007). Midterm outcomes of paclitaxel-eluting stents for the treatment of intracranial posterior circulation stenosis. J. Neurosurg. 106, 222-225.

Sundt, T. M., Jr, Smith, H. C., Campbell, J. K., Vlietstra, R. E., Cucchiara, R. F., Stanson, and A. W. (1980) Transluminal angioplasty for basilar artery stenosis. Mayo Clin. Proc. 55, 673-680.

Suri, F. K. M., and Johnston, C. S. (2009). Epidemiology of intracranial stenosis. J. Neuroimaging 19, 11S-16S.

Suwanwela, N. C., Chutinet, A., and Phanthumchinda, K. (2006). Inflammatory markers and conventional atherosclerotic risk factors in acute ischemic stroke: comparative study between vascular disease subtypes. J. Med. Assoc. Thai. 89, 2021-2027.

Takis, C., Kwan, E. S., Pessin, M. S., Jacobs, D. H., and Caplan, L. R. (1997). Intracranial angioplasty: experience and complications. Am. J. Neuroradiol. $18,1661-1668$.

Terada, T., Higashida, R. T., Halbach, V. V., Dowd, C. F., Nakai, E., Yokote, H., Itakura, T., and Hieshima, G. B. (1996). Transluminal angioplasty for 
arteriosclerotic disease of the distal vertebral and basilar arteries. J. Neurol. Neurosurg. Psychiatry 60, 377-381.

Turan, T.N., Maidan, L., Cotsonis, G., Lynn, M. J., Romano, J. G., Levine, S. R., and Chimowitz, M. I. (2009). Failure of antithrombotic therapy and risk of stroke in patients with symptomatic intracranial stenosis. Stroke 40, 505-509.

Volk, E. E., Prayson, R. A., and Perl, J. (1997). Autopsy finding of fatal complications of posterior cerebral circulation angioplasty. Arch. Pathol. Lab. Med. 121, 738-740.

White, H., Boden-Albala, B., Wang, C., Elkind,M.S., Rundek, T., Wright, C. B., and Sacco, R. L. (2005). Ischemic stroke subtype incidence among whites, blacks, and Hispanics: the Northern Manhattan Study. Circulation 111, 1327-1331.

Wong, K. S. (2003). Long-term mortality and recurrent stroke risks among Chinese stroke patients with predominant intracranial atherosclerosis. Stroke 34, 2361-2366.

Wong, K. S., Huang, Y. N., Gao, S., Lam, W. W., Chan, Y. L., and Kay, R. (1998). Intracranial stenosis in Chinese patients with acute stroke. Neurology 50, 812.

Zaidat, O. O., Klucznik, R., Alexander, M. J., Chaloupka, J., Lutsep, H., Barnwell,
S., Mawad, M., Lane, B., Lynn, M. J., and Chimowitz, M. (2008). The NIH registry on the use of the Wingspan stent for symptomatic 70-99\% intracranial arterial stenosis. Neurology 70 , 1518-1524.

Conflict of Interest Statement: The authors declare that the research was conducted in the absence of any commercial or financial relationships that could be construed as a potential conflict of interest.

Received: 10 August 2010; accepted: 27 December 2010; published online: 14 February 2011.
Citation: Short JL, Majid A and Hussain SI (2011) Endovascular treatment of symptomatic intracranial atherosclerotic disease. Front. Neur. 1:160. doi: 10.3389/ fneur.2010.00160

This article was submitted to Frontiers in Endovascular and Interventional Neurology, a specialty of Frontiers in Neurology. Copyright (c) 2011 Short, Majid and Hussain. This is an open-access article subject to an exclusive license agreement between the authors and Frontiers Media $S A$, which permits unrestricted use, distribution, and reproduction in any medium, provided the original authors and source are credited. 